\title{
Safety parameters of Ferromagnetic Device during Thyroid Surgery: Porcine model using continuous neuromonitoring
}

\begin{tabular}{|c|c|}
\hline Journal: & Head \& Neck \\
\hline Manuscript ID & HED-20-0303.R1 \\
\hline Wiley - Manuscript type: & Original Article \\
\hline $\begin{array}{r}\text { Date Submitted by the } \\
\text { Author: }\end{array}$ & 28-Apr-2020 \\
\hline Complete List of Authors: & $\begin{array}{l}\text { Huang, Tzu-Yen; Kaohsiung Medical University Hospital, Otolaryngology- } \\
\text { Head and Neck Surgery } \\
\text { Lin, Yi-Chu; Kaohsiung Medical University Hospital, Kaohsiung Medical } \\
\text { University, Kaohsiung, Taiwan., Otolaryngology- Head and Neck Surgery } \\
\text { Tseng, Hsin-Yi; Kaohsiung Medical University } \\
\text { Dionigi, MD, FACS, Prof. Gianlorenzo; Universita degli Studi di Messina, } \\
\text { Kim, Hoon Yub; Korea University College of Medicine and School of } \\
\text { Medicine, Department of Surgery } \\
\text { Chai, Young Jun; Seoul National University Hospital and College of } \\
\text { Medicine, Department of Surgery; Seoul National University College of } \\
\text { Medicine, } \\
\text { Lu, I-Cheng; Kaohsiung Medical University Hospital, Department of } \\
\text { Anesthesiology } \\
\text { Chang, Pi-Ying; Kaohsiung Medical University Hospital, Anesthesiology } \\
\text { Chiang, Feng-Yu; Kaohsiung Medical University Hospital, Department of } \\
\text { Otolaryngology - Head and Neck Surgery, Kaohsiung Medical University; } \\
\text { Wu, Che-Wei; Kaohsiung Medical University, Department of } \\
\text { Otolaryngology-Head and Neck Surgery }\end{array}$ \\
\hline Key Words: & $\begin{array}{l}\text { Recurrent laryngeal nerve, Ferromagnetic device, Continuous } \\
\text { intraoperative neuromonitoring, Thyroid surgery, Nerve thermal injury }\end{array}$ \\
\hline
\end{tabular}

\section{SCHOLARONE Manuscripts}




\section{Safety parameters of Ferromagnetic Device during Thyroid Surgery: Porcine model using continuous neuromonitoring}

Tzu-Yen Huang ${ }^{1,2}$, Yi-Chu Lin ${ }^{1}$, Hsin-Yi Tseng ${ }^{1}$, Gianlorenzo Dionigi ${ }^{3}$, Hoon Yub $\mathrm{Kim}^{4}$, Young Jun Chai ${ }^{5}$, I-Cheng Lu ${ }^{6}$, Pi-Ying Chang ${ }^{6}$, Feng-Yu Chiang ${ }^{7,8}$, Che-Wei $\mathrm{Wu}^{1,9}$

${ }^{1}$ Department of Otolaryngology-Head and Neck Surgery, Kaohsiung Medical University Hospital, Kaohsiung Medical University, Kaohsiung, Taiwan

${ }^{2}$ Department of Biological Science and Technology, National Chiao Tung University, Taiwan

${ }^{3}$ Division for Endocrine and Minimally Invasive Surgery, Department of Human Pathology in Adulthood and Childhood "Gaetano Barresi", University Hospital G. Martino, University of Messina, Messina, Italy ${ }^{4}$ Department of Surgery, KUMC Thyroid Center, Korea University College of Medicine, Seoul, Korea ${ }^{5}$ Department of Surgery, Seoul Metropolitan Government-Seoul National University Boramae Medical Center, Seoul, Korea

${ }^{6}$ Department of Anesthesiology, Kaohsiung Municipal Siaogang Hospital, Kaohsiung Medical University Hospital, Faculty of Medicine, College of Medicine, Kaohsiung Medical University, Kaohsiung, Taiwan

${ }^{7}$ School of Medicine, College of Medicine, I-Shou University, Kaohsiung, Taiwan

${ }^{8}$ Department of Otolaryngology, E-Da Hospital, Kaohsiung, Taiwan

${ }^{9}$ Department of Otorhinolaryngology-Head and Neck Surgery, Kaohsiung Municipal Siaogang Hospital, Faculty of Medicine, College of Medicine, Kaohsiung Medical University, Kaohsiung, Taiwan

The authors have no funding, financial relationships, or conflicts of interest to disclose.

Send correspondence to Che-Wei $\mathrm{Wu}, \mathrm{MD}, \mathrm{PhD}$, Department of OtolaryngologyHead and Neck Surgery, Kaohsiung Medical University Hospital, 100 Tzyou First Road, Kaohsiung 807, Taiwan

E-mail: kmuent@yahoo.com.tw, cwwu@kmu.edu.tw 


\begin{abstract}
Background The ferromagnetic (FM) device is a new surgical energy modality. This study investigated dynamic recurrent laryngeal nerve (RLN) electromyographic (EMG) data to define safety parameters for using FM devices in thyroidectomy. Methods Real-time EMG tracings obtained under continuous neuromonitoring were recorded from 24 RLNs (12 piglets). In the activation study, FM devices were activated at varying distances from the RLN. In the cooling study, FM devices were cooled for varying time intervals, or muscle touch maneuver was performed, before contact with the RLN.

Results During the FMwand/FMsealer activation, no adverse EMG events occurred at distances of $2 \mathrm{~mm}$ or longer. The cooling study revealed no adverse EMG events after 1-second (FMwand) or 3-second (FMsealer) intervals or after muscle touch maneuver.
\end{abstract}

Conclusions An FM device should be activated at a distance of $2 \mathrm{~mm}$ from the RLN and should be adequately cooled before further contact with the RLN. Surgeons can avoid RLN injury by observing standard procedures for using FM devices.

Key Words: Recurrent laryngeal nerve, Ferromagnetic device, Continuous intraoperative neuromonitoring, Thyroid surgery, Nerve thermal injury. 


\section{Main Text \\ Introduction}

Surgical management of thyroid disease has evolved considerably in the past several decades. In recent years, research and development of energy-based devices (EBD) have played an important role in improving the overall quality of thyroid surgery. Use of EBDs in thyroid surgery has increased because EBDs increase hemostasis efficiency, decrease operation time, and prevent life-threatening hematoma ${ }^{1-3}$. Some studies indicate that, compared to conventional clamp-and-tie technique, EBDs obtain superior outcomes in thyroid surgery, including pain, hospital stay, blood loss, postoperative drainage, and incidence of postoperative hypocalcemia.

${ }^{4-7}$ However, the high temperatures, lateral thermal spread, and the residual heat generated by EBDs can cause unexpected iatrogenic thermal injury to recurrent laryngeal nerves (RLNs). ${ }^{8,9}$ Therefore, surgeons should follow standard procedures and understand safety parameters when using EBDs for hemostasis and dissection in thyroid surgery.

Currently, the EBDs most commonly used in thyroid surgery are based on bipolar (Ligasure) and ultrasonic (Harmonic) technology. Each method has inherent advantages and disadvantages resulting from their different mechanisms. ${ }^{1,3}$

Ferromagnetic (FM) technology is a new surgical EBD that could be effective for hemostasis and dissection during thyroid surgery. This device generates highfrequency alternating current and transmits it through the heating elements of the device to create a rapidly alternating magnetic field. The FM alloy responds to the rapidly alternating magnetic field by immediately generating pure thermal energy through a combination of ohmic and hysteresis losses. Contact between the heating element of the FM device and the tissue minimizes lateral thermal spread to surrounding tissue and achieves a hemostatic effect. ${ }^{10,11}$ The device reportedly maintains electrical silence in terms of muscle contraction, nerve stimulation and interference with electrically sensitive devices, e.g., pacemakers or automatic internal defibrillators. ${ }^{12}$

The FMwand (Domain Surgical, Salt Lake City, UT, USA) is a commercially available hemostatic dissecting scalpel with a dissecting loop that uses FM heating as an energy source. ${ }^{13}$ Radiofrequency current from a generator is conducted through an alloy loop and returned to ground without passing through the patient. The loop has a ferromagnetic coating several microns in thickness and is coupled to the high frequency alternating current. (Fig. 1A).

The FMsealer (Domain Surgical, Salt Lake City, UT, USA) is an open shear consisting of a FM heating element in the jaw of a surgical vessel sealing device. Compressing tissue with the open shear generates thermal energy on the heating 
element from the active jaw to the inactive jaw. Thermally, the tissue is relatively inert. Heat transmitted perpendicularly through the compressed tissue seals and divides the tissue and vessels without additional cutting mechanisms. In vitro experiments have confirmed that FMsealer effectively seals arteries and veins with diameters ranging from 1 to $7 \mathrm{~mm}$ (as measured in a noncompressed naturally pressurized state) with burst pressures consistent with BP sealers and equivalent reliability of vessel seals. ${ }^{13}$ (Fig. 1B)

No studies have published experimental or clinical data regarding the safety of FM device in relation to hemostasis and dissection near the RLN during thyroidectomy. The purpose of this study was to investigate real-time electromyographic (EMG) data for the RLN to define safety parameters for using an FM device in thyroidectomy. For this purpose, we recorded the dynamic evoked laryngeal EMG change under continuous intraoperative neuromonitoring (CIONM) when the FM device was used at various distances from the RLN (activation study). We also evaluated the effects of various cooling durations and cooling by muscle touch maneuver to determine the optimal post-activation parameters for cooling an FM device (cooling study).

\section{MATERIALS AND METHODS}

\section{Subject Preparation and Anesthesia}

The prospective porcine model used for the CIONM experiments in this study was developed by a research team at Kaohsiung Medical University, Taiwan ${ }^{14,15}$. The animal-use protocol was consistent with national/international regulations and guidelines for animal experiments, including replacement, reduction, and refinement principles, and was approved by the Institutional Animal Care and Use Committee.

General anesthesia in the experiment was induced by intravenous injection of thiopental $15 \mathrm{mg} / \mathrm{kg}$ with the animal in a supine position. Endotracheal surface electrodes were intubated to record EMG signals generated by electrical stimulation applied from the RLN to the vocalis muscles. Neuromuscular blockade that might interfere with EMG signals under IONM was avoided by excluding use of muscle relaxants throughout the anesthesia process. After the piglets were intubated, tidal volume was set to $8 \mathrm{~mL} / \mathrm{kg}$, and respiratory rate was set to 15 to 20 breaths per minute. General anesthesia was maintained with sevoflurane $1 \%$ to $2 \% .{ }^{15}$

\section{Equipment Setting and Operation}

All IONM procedures were performed according to International Neural Monitoring Study Group guidelines. ${ }^{16}$ A nerve integrity monitor size \#6 EMG endotracheal tube (NIM Standard Tube, Medtronic, Jacksonville, FL, USA) was used in the manner routinely applied in humans. The CIONM was performed using the 


\section{Study Design}

The activation study determined the distance from the RLN at which the FM devices can be safely used. The cooling study determined the time intervals and procedures required to cool an FM device before further use in hemostasis and dissection near the RLN. Figure 1 shows the FMwand, FMsealer and the FMX G1 Generator system (Domain Surgical, Salt Lake City, UT, USA) used in this study. In this experiment, the activation power of FMwand was set at 45, and the activation power of FMsealer was set at 3.-

Activation study. Figures 2-3 depict the designs of the FMwand activation study and the FMsealer activation study, respectively. The FMwand and FMsealer were applied to soft tissue in a single activation of 3 seconds and at a distance of $5 \mathrm{~mm}$ from the RLN. If no adverse EMG event (e.g., significant adverse decrease in amplitude or increase in latency) occurred, the distance was progressively decreased to $2 \mathrm{~mm}$ and then to $1 \mathrm{~mm}$. Tests proceeded from the proximal to the distal part of the RLN. Realtime EMG CIONM information was continuously recorded during each FM device activation. A substantial adverse EMG change was interpreted as an injury on the RLN side. Dynamic EMG changes were continuously recorded for at least 20 minutes to observe any recovery of the electrophysiological response.

Cooling study. Figures 4-5 show the designs of the cooling studies for FMwand and FMsealer, respectively. First, the FM devices were applied to the SCM muscle in a 
single activation of 3 seconds then allowed to cool at room temperature for 5 seconds. The dissecting loop blade of the FMwand or the open shear of the FMsealer was then applied to the RLN. If no adverse EMG event occurred, cooling time was decreased to 3 seconds and then immediately (estimated 1-second time interval for shifting the device from the muscle to the RLN). To test the cooling effect of the "muscle touch maneuver", the operator activated the FM device on the SCM muscle and then touched another part of the SCM muscle. The operator then allowed the FM device to cool for varying time intervals before touching the RLN. Muscle touch maneuver was performed after a single activation of the FM device on the SCM muscle. With the heating element of the device in full contact with the SCM muscle at another position, the device was immediately withdrawn from the muscle and placed in contact with RLN. Real-time EMG information for each stimulation was recorded and analyzed. When an RLN injury occurred, dynamic EMG changes were continuously recorded for at least 20 minutes to observe any recovery.

\section{Results}

The animal anesthesia, surgical approach, and CIONM were successfully performed in all experiments. Tables 1-2 summarize the results of the FMwand and FMsealer activation and cooling experiments.

\section{Activation Study}

In tests of FMwand activation at distances of 5 and $2 \mathrm{~mm}$, no adverse effects, including amplitude decreases and prolonged latency, occurred. With the shear activated at a distance of $1 \mathrm{~mm}$, all RLNs of piglets showed loss of signal (LOS) after a 20-minute observation (Table 1 and Fig. 2C). In tests of FMsealer activation at distances of 5 and $2 \mathrm{~mm}$, no adverse effects, including amplitude decreases and prolonged latency, occurred. With the shear activated at distance of $1 \mathrm{~mm}$, all RLNs of piglets showed at least $71.2 \%$ LOS within a 20 -minute observation period (Table1). The left RLN in piglet 6 showed LOS without recovery (Fig. 3C).

\section{Cooling Study}

Table 2 shows the details of the cooling study performed in twelve RLNs of six piglets. After a 3-second activation of FMwand followed by a cooling time of 5 seconds, 3 seconds, or immediately, no piglets showed adverse effects when the RLN was touched with the dissecting loop, regardless of whether muscle touch maneuver was performed (Table 2 and Fig. 4C).

When FMsealer was activated for 3 seconds and then allowed to cool for 5 seconds or for 3 seconds, neither side of the RLN showed adverse EMG events when the RLN 
was touched with the shear (Table 2). When FMsealer was activated for 3 seconds and then immediately placed in contact with the RLN, three of six nerves showed at least 58.3\% EMG signal loss without recovery over a 20 -minute observation time (Fig. 5C). In the remaining three RLNs, no adverse effects occurred when the muscle touch maneuver was performed after 3 seconds cooling time or immediately (Table 2).

\section{Discussion}

To our best knowledge, this is the first study offered a standardized manner for surgeons to use FM devices near the RLN in thyroid surgery. In the activation study, there was no adverse EMG events occurred when the FMwand and FMsealer were activated at a distance of $2 \mathrm{~mm}$ or longer from the RLN. In the cooling study, no adverse EMG events occurred after 1-second (FMwand) and 3-second (FMsealer) time intervals or after cooling by muscle touch maneuver.

A thermal injury to the RLN caused by an EBD is of moderate to high severity because recovery is usually poor and because histological disturbance is usually severe relative to other injuries such as traction injury and mechanical trauma ${ }^{8}$. A thermal injury may be difficult to recognize visually, and lateral thermal spread can occur without direct contact with the heat source. ${ }^{20,21}$ Application of spread stimuli over $60^{\circ} \mathrm{C}$ to the RLN can cause permanent functional damage to the endoneurium ${ }^{22}$ whereas traction injury is usually limited to the perineurium and epineurium. ${ }^{8,17}$ After activation for use in thyroid surgery, most EBDs can reach temperatures higher than $60^{\circ} \mathrm{C}$, which can potentially cause RLN thermal injuries of varying severity. No studies have reported experimental or clinical evidence of the safety of FM devices in terms of hemostasis and dissection near the RLN during thyroidectomy. Hence, this study investigated the thermal effects of FM devices to determine their safety parameters during thyroid surgery.

Intraoperative neural monitoring has gained widespread acceptance as an adjunct to the gold standard of visual identification of the RLN during thyroid surgery ${ }^{23,24}$. Use of CIONM can improve thyroid surgery outcomes by detecting anatomic variations that can cause injuries ${ }^{25-27}$ and by detecting the presence and mechanisms of nerve invasion or injuries that are predictive of postoperative vocal fold function. ${ }^{24,28-31}$ Use of APS during CIONM provides visual and acoustic feedback that thyroid surgeons can use to evaluate nerve function and to facilitate early identification of nerve injury mechanisms, especially traction injuries. In high-risk procedures, CIONM enables the operator to take corrective action to maximize preservation of nerve function. ${ }^{18,24}$ However, thermal injury can occur suddenly and unexpectedly when an EBD is used in thyroid surgery, and recovery from such 
injuries is often difficult. Therefore, taking precautions to avoid injuries caused by EBDs during surgery is more important than using CIONM to identify thermal injuries.

Recently developed FM devices substantially increase the precision of surgical dissection in thyroid surgery. The FMwand enables rapid and precise control of heating and cooling. The generated heat is restricted to the coating itself and the tissue in contact with the coating. ${ }^{10,11}$ Since no electrical energy or magnetic effects are applied to surrounding tissue, the device does not interfere with electrophysiological monitors, pacemakers or automatic internal defibrillators. The FMsealer consists of an FM heating element in the jaw of a surgical vessel sealing device. Chen et al. reported several features of the FMsealer ${ }^{32}$. The authors evaluated the reliability of FMsealer by using it to seal porcine arteries (diameter, $5-7 \mathrm{~mm}$ ) in a controlled laboratory setting. A 95\% success rate was achieved for a burst pressure above $240 \mathrm{mg}$. Each vessel was sealed, divided and pressurized using an automated inflation system until the seal failed. The average burst pressure was $1098 \mathrm{mmHg}$. In a blinded histologic evaluation of $5 \mathrm{~mm}$ vessel seals in vivo, the distance of lateral thermal spreads for the FM $(1678 \pm 433 \mu \mathrm{m})$ and Ligasure $(1796 \pm 337 \mu \mathrm{m})$ devices were significantly shorter compared to that for the Harmonic device $(2032 \pm 387 \mu \mathrm{m}), \mathrm{P}<0.001$.

EBDs significantly improved efficacy in achieving hemostasis and were widely used in thyroid surgery. Several porcine model studies investigated the commonly used hemostatic devices and their safety parameters when dissecting RLN ${ }^{33}$. The safe activation distances and cooling times were $5 \mathrm{~mm}$ and 1 second for monopolar electrocautery ( 15 watts); $3 \mathrm{~mm}$ and 1 second for bipolar electrocautery $\left(30\right.$ watts) ${ }^{34}$; $2 \mathrm{~mm}$ and 2 seconds (or cooled by touch muscle maneuver) for the Ligasure Small $\mathrm{Jaw}^{35} ; 1 \mathrm{~mm}$ and 2 seconds (or cooled by touch muscle maneuver) for the Ligasure Exact Dissector ${ }^{36} ; 2 \mathrm{~mm}$ and 10 seconds (or cooled by touch muscle maneuver for 2 seconds) for the Harmonic Focus ${ }^{37} ; 4 \mathrm{~mm}$ for the Harmonic ACE, and $1 \mathrm{~mm}$ for the Harmonic $\mathrm{ACE}+38 ; 3 \mathrm{~mm}$ for the THUNDERBEAT ${ }^{3936}$. The current study investigates the safety parameters of FMs and confirms the FMs are comparable to those commonly used hemostatic devices, and thus FMs can be a potential alternative EBD to facilitate efficient hemostasis and dissection during thyroid surgery.

Since the FMwand and FMsealer share the same power module, the module can be switched during surgery. Therefore, one power module can be used in different dissection stages in thyroid surgery. The thin non-stick dissection loop of the FMwand (Figs. 1A, 2B, and 4B) can simultaneously cut and coagulate soft tissue while minimizing collateral tissue damage. Therefore, the FMwand is useful for skin flap elevation and precise dissection in the Berry ligament region or for 
dissection of the parathyroid glands. The FMsealer Open Shears (Figs. 1B, 3B, and 5B) provides reliable vessel sealing and minimal thermal impact on healthy tissue and is effective for hemostasis of thyroid vessels and dissection near the RLN.

This study had the following limitations. First, the data and information obtained from this animal study may require further verification in human surgery. the data and information obtained from a prospective porcine model animal study may not beapplicable to human surgery. Many factors can affect thermal transfer from FM devices to the RLN, such as the surgical environment and the use of different species for animal experiments. However, this model has proven useful and reliable for depicting real-time changes in laryngeal EMG caused by RLN injury. ${ }^{8,15,17,20-22}$. A second limitation is that this study only depicted real-time EMG changes during a 20minute period rather than long-term changes in RLN function. Notably, the use of CIONM data for predicting vocal cord function is well established. ${ }^{18,40}$ Finally, when applied to actual human surgery, soft tissue coagulation may shorten the distance from the FM device to the RLN. Therefore, although this study indicates that the minimum safe distance for the FMwand and FMsealer is $2 \mathrm{~mm}$, the tip should always be carefully inspected during activation and RLN dissection should be stopped immediately if soft tissue coagulation tends to draw the nerve toward the tip of the device.

\section{CONCLUSION}

This study used real-time laryngeal EMG data to define safety parameters for using an FM device near the RLN. Ensuring a sufficient activation distance of FM devices is mandatory. Therefore, whenever the FM devices were activated, the RLN should be clearly visible and maintain a safe distance. After activation, the device should be cooled before further use in any procedure that requires direct contact with the RLN. Knowing their respective safety parameters helps to avoid RLN thermal injury and increases surgical efficiency during thyroid surgery.

\section{ACKNOWLEDGEMENTS}

This study was supported by grants from Kaohsiung Medical University Hospital, Kaohsiung Medical University (KMUH107-7R50, KMUH108-8M48, KMUH SA10807c), Kaohsiung Municipal Siaogang Hospital, Kaohsiung Medical University (H-108-005), and from Ministry of Science and Technology (MOST 108-2628-B037-006), Taiwan

\section{Reference}

1. Cannizzaro MA, Borzi L, Lo Bianco S, Okatyeva V, Cavallaro A, Buffone A. 
Comparison between Focus Harmonic scalpel and other hemostatic techniques in open thyroidectomy: A systematic review and meta-analysis. Head \& neck. 2016;38(10):1571-1578.

2. Patel KN, Yip L, Lubitz CC, et al. The American Association of Endocrine Surgeons Guidelines for the Definitive Surgical Management of Thyroid Disease in Adults. Annals of surgery. 2020;271(3):e21-e93.

3. Bakkar S, Papavramidis TS, Aljarrah Q, Materazzi G, Miccoli P. Energy-based devices in thyroid surgery-an overview. Gland surgery. 2020;9(Suppl 1):S14s17.

4. Chiang FY, Lee KD, Tae K, et al. Comparison of hypocalcemia rates between LigaSure and clamp-and-tie hemostatic technique in total thyroidectomies. Head \& neck. 2019;41(10):3677-3683.

5. Papavramidis TS, Sapalidis K, Michalopoulos N, et al. UltraCision harmonic scalpel versus clamp-and-tie total thyroidectomy: a clinical trial. Head \& neck. 2010;32(6):723-727.

6. Melck AL, Wiseman SM. Harmonic scalpel compared to conventional hemostasis in thyroid surgery: a meta-analysis of randomized clinical trials. Int J Surg Oncol. 2010;2010:396079.

7. Duan YF, Xue W, Zhu F, Sun DL. FOCUS harmonic scalpel compared to conventional hemostasis in open total thyroidectomy - a prospective randomized study. Journal of otolaryngology - head \& neck surgery = Le Journal d'oto-rhino-laryngologie et de chirurgie cervico-faciale. 2013;42:62.

8. Dionigi G, Wu CW, Kim HY, Rausei S, Boni L, Chiang FY. Severity of Recurrent Laryngeal Nerve Injuries in Thyroid Surgery. World journal of surgery. 2016;40(6):1373-1381.

9. Dionigi G, Wu CW, Kim HY, et al. Safety of energy based devices for hemostasis in thyroid surgery. Gland surgery. 2016;5(5):490-494.

10. Bowers CA, Burns G, Salzman KL, McGill LD, MacDonald JD. Comparison of tissue effects in rabbit muscle of surgical dissection devices. International Journal of Surgery. 2014;12(3):219-223.

11. MacDonald JD, Bowers CA, Chin SS, Burns G. Comparison of the effects of surgical dissection devices on the rabbit liver. Surgery today. 2014;44(6):1116-1122.

12. Weiss JP, Manwaring P. Freedom from electromagnetic interference between cardiac implantable electronic devices and the FMwand ferromagnetic surgical system. Journal of clinical anesthesia. 2013;8(25):681-684.

13. Chen J, Manwaring PK, Scott RR, Manwaring KH, Glasgow RE. Ferromagnetic heating for vessel sealing and division: utility and comparative study to 
ultrasonic and bipolar technologies. Surgical innovation. 2015;22(4):329-337.

14. Wu CW, Lu IC, Randolph GW, et al. Investigation of optimal intensity and safety of electrical nerve stimulation during intraoperative neuromonitoring of the recurrent laryngeal nerve: a prospective porcine model. Head \& neck. 2010;32(10):1295-1301.

15. Wu CW, Huang TY, Chen HC, et al. Intra-Operative Neural Monitoring of Thyroid Surgery in a Porcine Model. Journal of visualized experiments : JoVE. 2019(144):doi: 10.3791/57919.

16. Randolph GW, Dralle $\mathrm{H}$, International Intraoperative Monitoring Study $\mathrm{G}$, et al. Electrophysiologic recurrent laryngeal nerve monitoring during thyroid and parathyroid surgery: international standards guideline statement. Laryngoscope. 2011;121 Suppl 1:S1-16.

17. Wu CW, Dionigi G, Sun $\mathrm{H}$, et al. Intraoperative neuromonitoring for the early detection and prevention of RLN traction injury in thyroid surgery: A porcine model. Surgery. 2014;155(2):329-339.

18. Schneider R, Sekulla C, Machens A, Lorenz K, Thanh PN, Dralle H. Dynamics of loss and recovery of the nerve monitoring signal during thyroidectomy predict early postoperative vocal fold function. Head \& neck. 2016;38 Suppl 1:E1144-1151.

19. Brauckhoff K, Svendsen OS, Stangeland L, Biermann M, Aas T, Husby PJA. Injury mechanisms and electromyographic changes after injury of the recurrent laryngeal nerve: Experiments in a porcine model. Head \& neck. 2018;40(2):274-282.

20. Wu CW, Chai YJ, Dionigi G, et al. Recurrent laryngeal nerve safety parameters of the Harmonic Focus during thyroid surgery: Porcine model using continuous monitoring. Laryngoscope. 2015;125(12):2838-2845.

21. Dionigi G, Chiang FY, Kim HY, et al. Safety of LigaSure in recurrent laryngeal nerve dissection-porcine model using continuous monitoring. Laryngoscope. 2017;127(7):1724-1729.

22. Lin YC, Dionigi G, Randolph GW, et al. Electrophysiologic monitoring correlates of recurrent laryngeal nerve heat thermal injury in a porcine model. Laryngoscope. 2015;125(8):E283-290.

23. Randolph GW, Dralle $\mathrm{H}$, Abdullah $\mathrm{H}$, et al. Electrophysiologic recurrent laryngeal nerve monitoring during thyroid and parathyroid surgery: international standards guideline statement. Laryngoscope. 2011;121 Suppl 1:S1-16.

24. Fundakowski CE, Hales NW, Agrawal N, et al. Surgical management of the recurrent laryngeal nerve in thyroidectomy: American Head and Neck Society 
Consensus Statement. Head \& neck. 2018;40(4):663-675.

25. Chiang FY, Lu IC, Chen HC, et al. Anatomical variations of recurrent laryngeal nerve during thyroid surgery: how to identify and handle the variations with intraoperative neuromonitoring. Kaohsiung J Med Sci. 2010;26(11):575-583.

26. Kamani D, Potenza AS, Cernea CR, Kamani YV, Randolph GW. The nonrecurrent laryngeal nerve: anatomic and electrophysiologic algorithm for reliable identification. Laryngoscope. 2015;125(2):503-508.

27. Chiang FY, Lu IC, Tsai CJ, Hsiao PJ, Lee KW, Wu CW. Detecting and identifying nonrecurrent laryngeal nerve with the application of intraoperative neuromonitoring during thyroid and parathyroid operation. Am J Otolaryngol. 2012;33(1):1-5.

28. Chiang FY, Lu IC, Kuo WR, Lee KW, Chang NC, Wu CW. The mechanism of recurrent laryngeal nerve injury during thyroid surgery--the application of intraoperative neuromonitoring. Surgery. 2008;143(6):743-749.

29. Snyder SK, Lairmore TC, Hendricks JC, Roberts JW. Elucidating mechanisms of recurrent laryngeal nerve injury during thyroidectomy and parathyroidectomy. J Am Coll Surg. 2008;206(1):123-130.

30. Schneider R, Randolph GW, Dionigi G, et al. International neural monitoring study group guideline 2018 part I: Staging bilateral thyroid surgery with monitoring loss of signal. Laryngoscope. 2018;128 Suppl 3:S1-s17.

31. Wu CW, Dionigi G, Barczynski M, et al. International neuromonitoring study group guidelines 2018: Part II: Optimal recurrent laryngeal nerve management for invasive thyroid cancer-incorporation of surgical, laryngeal, and neural electrophysiologic data. Laryngoscope. 2018;128 Suppl 3:S18-s27.

32. Chen J, Jensen CR, Manwaring PK, Glasgow RE. Validation of a Laparoscopic Ferromagnetic Technology-based Vessel Sealing Device and Comparative Study to Ultrasonic and Bipolar Laparoscopic Devices. Surgical laparoscopy, endoscopy \& percutaneous techniques. 2017;27(2):e12.

33. Dionigi G, Wu C-W, Kim H-Y, et al. Safety of energy based devices for hemostasis in thyroid surgery. Gland surgery. 2016;5(5):490.

34. Wu C-W, Huang T-Y, Chen $\mathrm{H}-\mathrm{C}$, et al. Intra-Operative Neural Monitoring of Thyroid Surgery in a Porcine Model. JoVE (Journal of Visualized Experiments). 2019(144):e57919.

35. Dionigi G, Chiang FY, Kim HY, et al. Safety of LigaSure in recurrent laryngeal nerve dissection-porcine model using continuous monitoring. The Laryngoscope. 2017;127(7):1724-1729.

36. Huang $\mathrm{T}-\mathrm{Y}$, Lin $\mathrm{Y}-\mathrm{C}$, Tseng $\mathrm{H}-\mathrm{Y}$, et al. Safety of Ligasure exact dissector in thyroidectomy with continuous neuromonitoring: a porcine model. Gland 
surgery. 2020.

37. Wu CW, Chai YJ, Dionigi G, et al. Recurrent laryngeal nerve safety parameters of the $\mathrm{H}$ armonic $\mathrm{F}$ ocus during thyroid surgery: Porcine model using continuous monitoring. The Laryngoscope. 2015;125(12):2838-2845.

38. Kim HK, Chai YJ, Lee HY, Kim HY, Dionigi G. Comparing the safety of harmonic $A C E$ and $A C E+$ around the recurrent laryngeal nerve in swine models. Annals of surgical treatment and research. 2018;94(6):285-290.

39. Kwak HY, Dionigi G, Kim D, et al. Thermal injury of the recurrent laryngeal nerve by THUNDERBEAT during thyroid surgery: findings from continuous intraoperative neuromonitoring in a porcine model. journal of surgical research. 2016;200(1):177-182.

40. Phelan E, Schneider R, Lorenz K, et al. Continuous vagal IONM prevents recurrent laryngeal nerve paralysis by revealing initial EMG changes of impending neuropraxic injury: a prospective, multicenter study. Laryngoscope. 2014;124(6):1498-1505. 


\section{Legends for Figures}

Figure 1. Surgical approach and equipment settings. (A) Settings for the FMwand Dissector and the FMX Generator. The activation power was set at 45.- (B) Settings for the FMsealer Open Shears and the FMX Generator. The activation power was set at 3. (C) Setup for continuous intraoperative neuromonitoring system with Nerve Integrity Monitor (NIM 3.0) system. (D) For neck exposure, thyroid glands, vagus nerves, and recurrent laryngeal nerves were adequately exposed. Automated periodic stimulation (APS) electrode is applied on the vagus nerve.

Figure 2. Activation study of FMwand. (A) Flowchart of activation study protocols. Single activation was discontinued automatically for 3 seconds. Tests were performed from the proximal to distal segments of the RLN. The distance from the tip of the FMwand to the RLN was measured. In this study, the first test was performed at a distance of $5 \mathrm{~mm}$ from the fifth tracheal ring. If the EMG remained stable after three tests, another test was performed at a distance of $2 \mathrm{~mm}$. If the EMG remained stable after repeated tests at a distance of $2 \mathrm{~mm}$, safety was tested at a distance of $1 \mathrm{~mm}$. If a substantial EMG change was noted, the RLN experiment was complete, and EMG was continuously monitored for at least 20 minutes. (B) The FMwand was tested at a distance of $5 \mathrm{~mm}$ from the left RLN. (C) Left side of piglet 2. The FMwand was activated at distances of 5, 2, and $1 \mathrm{~mm}$. After activation at $1 \mathrm{~mm}$, real-time EMG showed sudden LOS without recovery during 20 minutes of continuous EMG recording.

Figure 3. Activation study of FMsealer. (A) Flowchart of activation study protocols. (B). The FMsealer was tested at a distance of $5 \mathrm{~mm}$ from the left RLN. (C) Left side of piglet 6 . The FMsealer was activated at distances of 5, 2, and $1 \mathrm{~mm}$. After activation at $1 \mathrm{~mm}$, real-time EMG showed sudden LOS without recovery during 20 minutes of continuous EMG recording.

Figure 4. Cooling study of FMwand. (A) Flowchart of cooling study protocols. The tests were performed on the RLN from the proximal to distal segments. After a single FMwand activation on the SCM muscle (white arrow), the RLN (red arrow) was touched with the tip after varying cooling times. The fifth tracheal ring was touched after a cooling time of 5 seconds. If EMG remained stable in three tests, a 3 -second cooling time was tested. If the EMG remained stable after repeated tests, safety was tested immediately (estimated 1 second time interval for shifting the device from muscle to the RLN) after touching the RLN after a single activation, with or without the touch maneuver (star sign, quick touching/cooling with surrounding 
tissue). (B) The tip of the FMwand was used to touch the left RLN at the fifth tracheal ring level with 5 seconds lag time after 5 seconds activation on the surrounding muscle (white arrow) (C) Left side of piglet 9. The cooling study was performed with 5 seconds and 3 seconds cooling time and immediately touch RLN without muscle touch maneuver, and after a single activation. The EMG results showed no adverse effects. The EMG signal remained stable in repeated tests.

Figure 5. Cooling study of FMsealer. (A) Flowchart of cooling study protocols. (B) The left RLN was touched at the fifth tracheal ring level with the open tip of the FMsealer with a 5 -second lag time after 5 seconds activation on the surrounding muscle (white arrow). (C) Left side of piglet 10 . The cooling study was performed with cooling times of 5 seconds and 3 seconds after a single activation. No adverse effects on EMG occurred. When the RLN was touched with the FMsealer immediately without muscle touch maneuver and after a single activation, a sudden loss of real-time EMG signal occurred. After 20 minutes of continuous EMG recording, a $58.3 \%$ signal loss occurred (from $1137 \mu \mathrm{V}$ to $474 \mu \mathrm{V}$ ). 


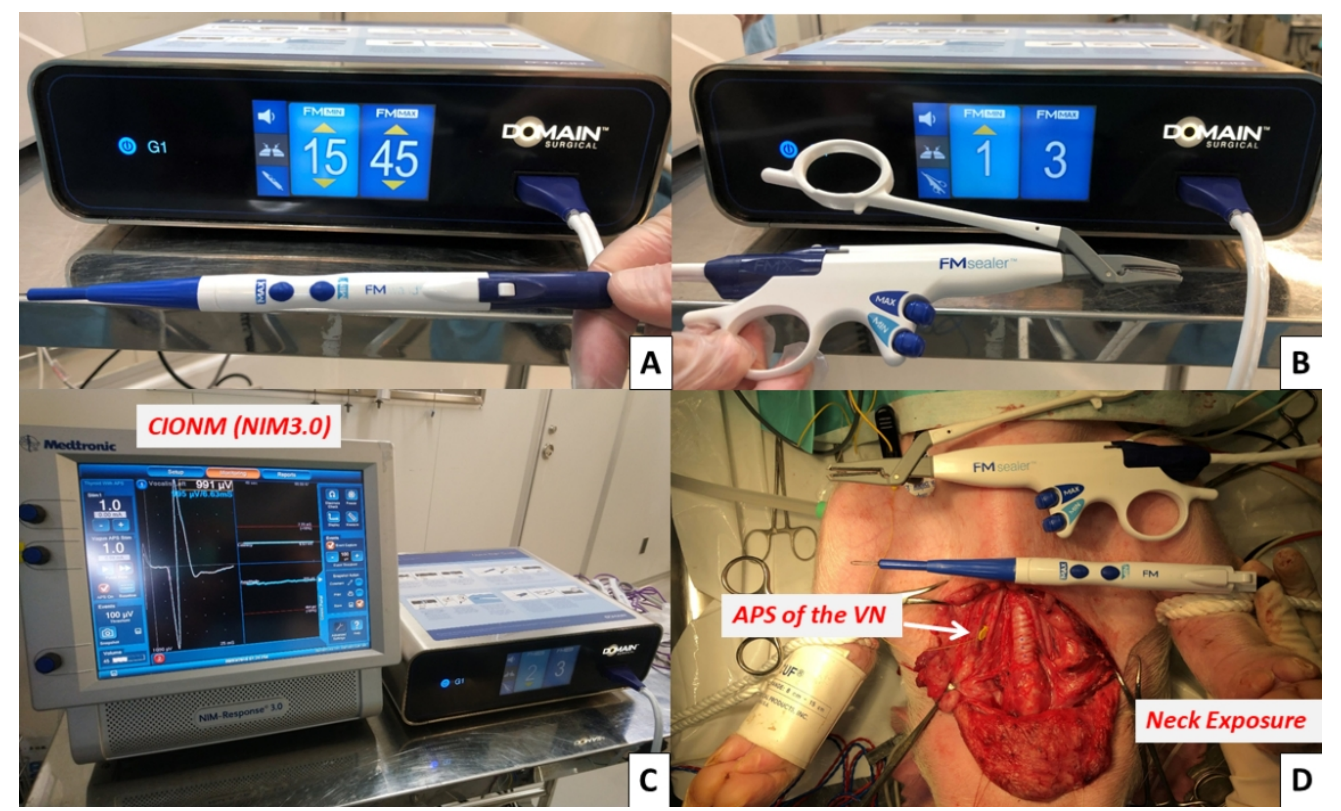

Figure 1. Surgical approach and equipment settings. (A) Settings for the FMwand Dissector and the FMX Generator. The activation power was set at 45. (B) Settings for the FMsealer Open Shears and the FMX Generator. The activation power was set at 3. (C) Setup for continuous intraoperative neuromonitoring system with Nerve Integrity Monitor (NIM 3.0) system. (D) For neck exposure, thyroid glands, vagus nerves, and recurrent laryngeal nerves were adequately exposed. Automated periodic stimulation (APS) electrode is applied on the vagus nerve. 


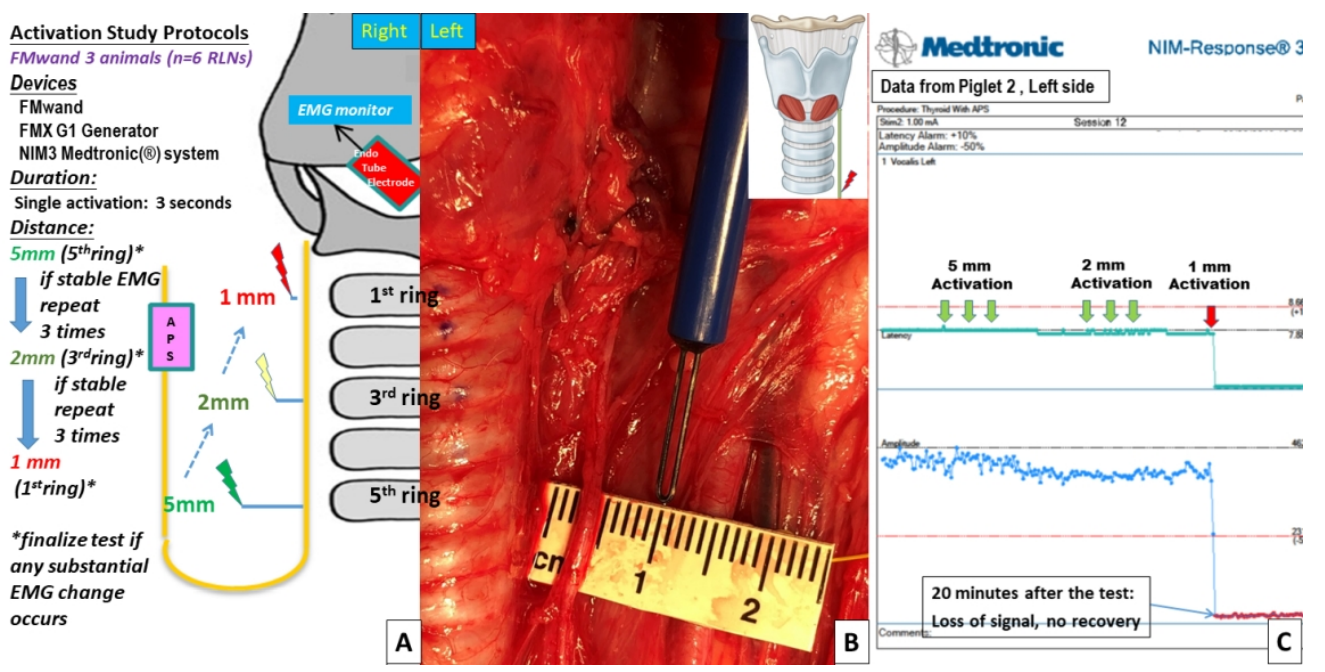

Figure 2. Activation study of FMwand. (A) Flowchart of activation study protocols. Single activation was discontinued automatically for 3 seconds. Tests were performed from the proximal to distal segments of the RLN. The distance from the tip of the FMwand to the RLN was measured. In this study, the first test was performed at a distance of $5 \mathrm{~mm}$ from the fifth tracheal ring. If the EMG remained stable after three tests, another test was performed at a distance of $2 \mathrm{~mm}$. If the EMG remained stable after repeated tests at a distance of $2 \mathrm{~mm}$, safety was tested at a distance of $1 \mathrm{~mm}$. If a substantial EMG change was noted, the RLN experiment was complete, and EMG was continuously monitored for at least 20 minutes. (B) The FMwand was tested at a distance of $5 \mathrm{~mm}$ from the left RLN. (C) Left side of piglet 2 . The FMwand was activated at distances of 5, 2, and $1 \mathrm{~mm}$. After activation at $1 \mathrm{~mm}$, real-time EMG showed sudden LOS without recovery during 20 minutes of continuous EMG recording. 


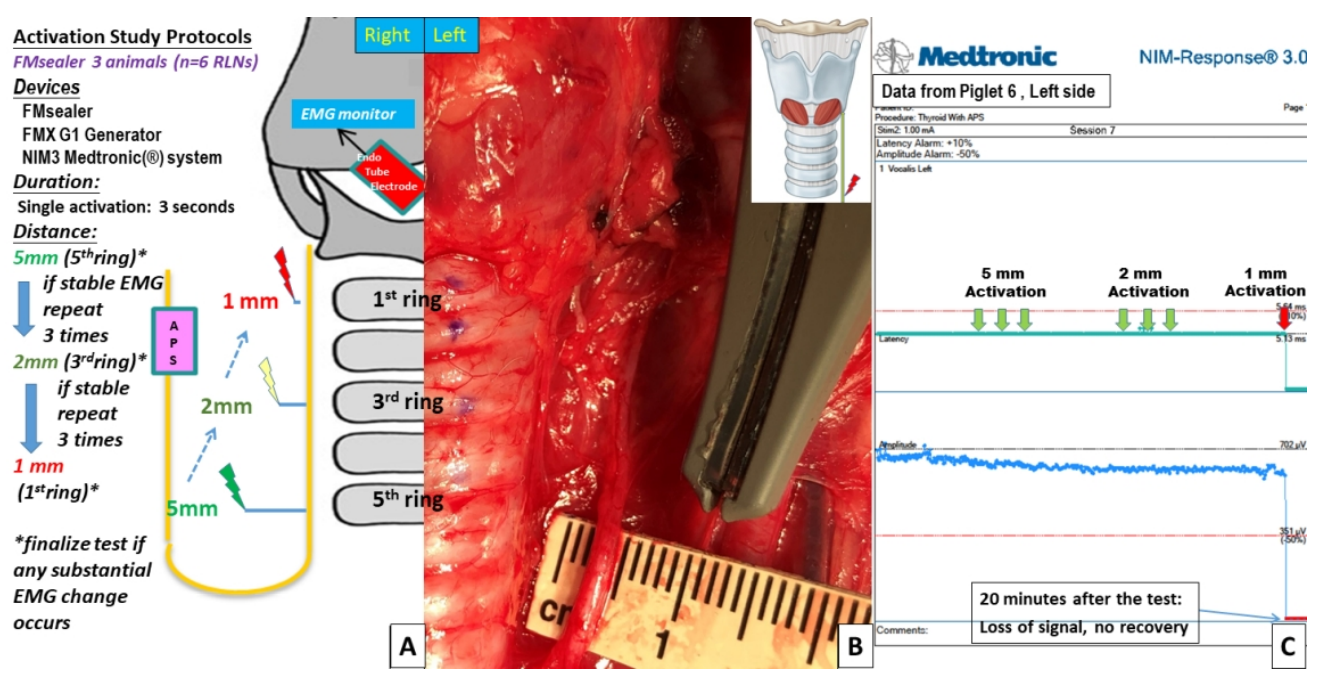

Figure 3. Activation study of FMsealer. (A) Flowchart of activation study protocols. (B). The FMsealer was tested at a distance of $5 \mathrm{~mm}$ from the left RLN. (C) Left side of piglet 6 . The FMsealer was activated at distances of 5,2 , and $1 \mathrm{~mm}$. After activation at $1 \mathrm{~mm}$, real-time EMG showed sudden LOS without recovery during 20 minutes of continuous EMG recording. 


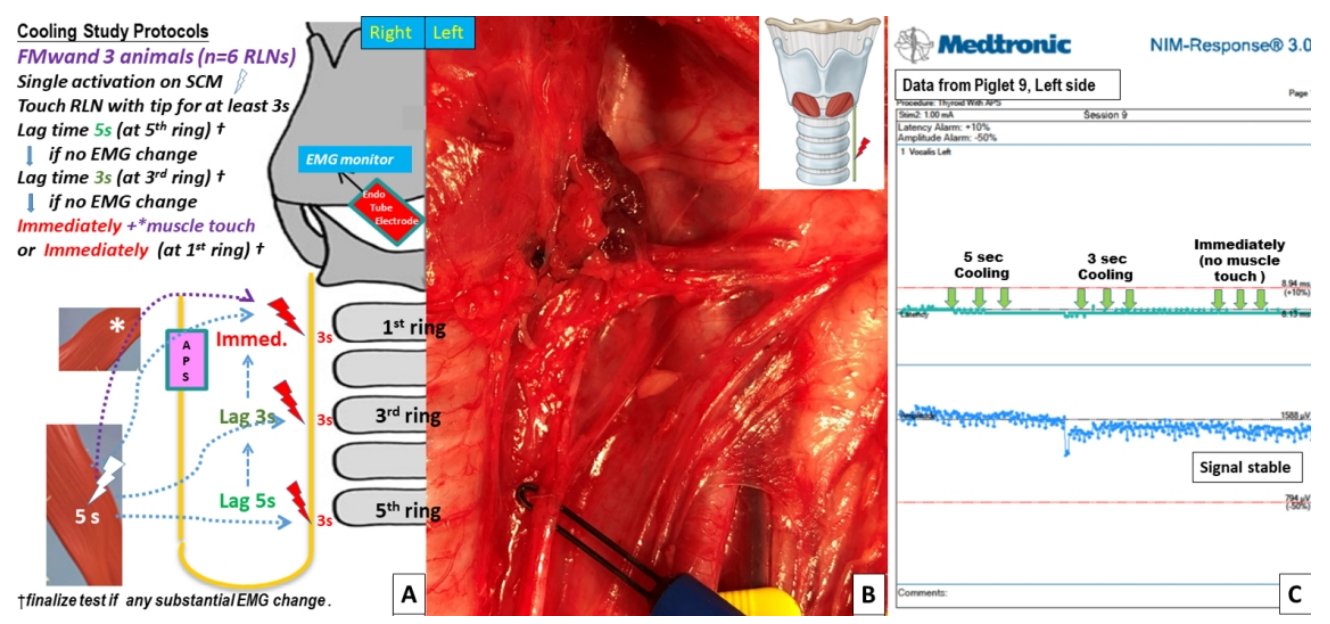

Figure 4. Cooling study of FMwand. (A) Flowchart of cooling study protocols. The tests were performed on the RLN from the proximal to distal segments. After a single FMwand activation on the SCM muscle (white arrow), the RLN (red arrow) was touched with the tip after varying cooling times. The fifth tracheal ring was touched after a cooling time of 5 seconds. If EMG remained stable in three tests, a 3-second cooling time was tested. If the EMG remained stable after repeated tests, safety was tested immediately (estimated 1 second time interval for shifting the device from muscle to the RLN) after touching the RLN after a single activation, with or without the touch maneuver (star sign, quick touching/cooling with surrounding tissue). (B) The tip of the FMwand was used to touch the left RLN at the fifth tracheal ring level with 5 seconds lag time after 5 seconds activation on the surrounding muscle (white arrow) (C) Left side of piglet 9 . The cooling study was performed with 5 seconds and 3 seconds cooling time and immediately touch RLN without muscle touch maneuver, and after a single activation. The EMG results showed no adverse effects. The EMG signal remained stable in repeated tests. 


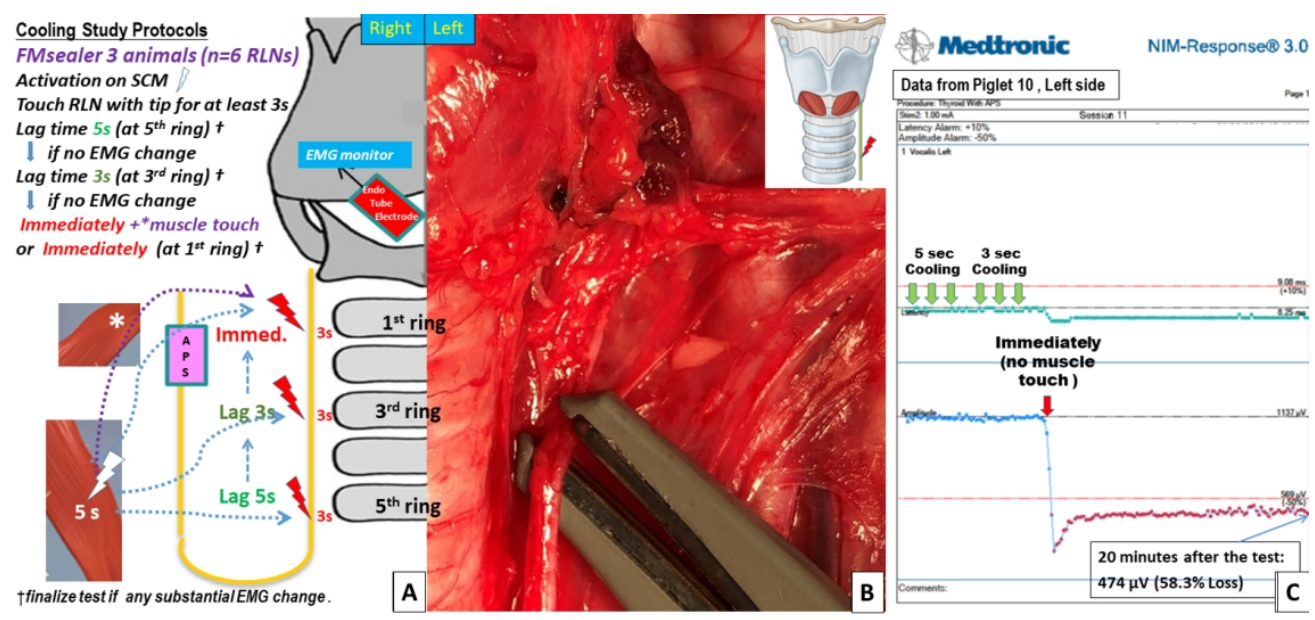

Figure 5. Cooling study of FMsealer. (A) Flowchart of cooling study protocols. (B) The left RLN was touched at the fifth tracheal ring level with the open tip of the FMsealer with a 5-second lag time after 5 seconds activation on the surrounding muscle (white arrow). (C) Left side of piglet 10 . The cooling study was performed with cooling times of 5 seconds and 3 seconds after a single activation. No adverse effects on EMG occurred. When the RLN was touched with the FMsealer immediately without muscle touch maneuver and after a single activation, a sudden loss of real-time EMG signal occurred. After 20 minutes of continuous EMG recording, a $58.3 \%$ signal loss occurred (from $1137 \mu \mathrm{V}$ to $474 \mu \mathrm{V}$ ). 
Table 1. Activation Study: Comparison of Real-Time EMG Change after Fmwand and FMsealer Activation at Varying Distances to RLN

\begin{tabular}{|c|c|c|c|c|}
\hline \multicolumn{2}{|c|}{ Animal } & \multicolumn{3}{|c|}{ Distance (EMG* [times of test]) } \\
\hline No. & $\overline{\text { Side }}$ & $5 \mathrm{~mm}$ & $2 \mathrm{~mm}$ & $1 \mathrm{~mm}$ \\
\hline \multicolumn{5}{|c|}{ FMwand } \\
\hline \multirow[t]{2}{*}{1} & $\mathrm{~L}$ & Stable [3] & Stable [3] & LOS' $^{+}[1]$ \\
\hline & $\mathrm{R}$ & Stable [3] & Stable [3] & LOS' $^{+}[1]$ \\
\hline \multirow[t]{2}{*}{2} & $\mathrm{~L}$ & Stable [3] & Stable [3] & $\operatorname{LOS}^{+}[1]$ \\
\hline & $\mathrm{R}$ & Stable [3] & Stable [3] & LOS' $^{+}[1]$ \\
\hline \multirow[t]{2}{*}{3} & $\mathrm{~L}$ & Stable [3] & Stable [3] & $\operatorname{LOS}^{+}[1]$ \\
\hline & $\mathrm{R}$ & Stable [3] & Stable [3] & LOS' $^{+}[1]$ \\
\hline
\end{tabular}

FMsealer

$4 \quad \mathrm{~L} \quad$ Stable [3] Stable [3] $71.2 \%$ loss [1]

$\mathrm{R} \quad$ Stable [3] Stable [3] $\quad$ LOS $^{\dagger}$ [1]

$5 \quad \mathrm{~L} \quad$ Stable [3] Stable [3] $77.4 \%$ loss [1]

R $\quad$ Stable [3] Stable [3] $\quad$ LOS $^{+}$[1]

$6 \quad \mathrm{~L} \quad$ Stable [3] $\quad$ Stable [3] $\quad$ LOS $^{+}$[1]

$\mathrm{R} \quad$ Stable [3] $\quad$ Stable [3] $\quad$ LOS $^{+}$[1]

* EMG, electromyography, data of unstable column represents the final amplitude after at least 20 minutes of continuously real-time EMG recording.

† LOS, loss of signal, defined as the evoked EMG amplitude less than $100 \mu \mathrm{V}$ 
Table 2. Cooling Study: Comparison of Real-Time EMG Change when Varying Cooling Time or Muscle Touch Maneuver was Performed after Fmwand and FMsealer Activation

\begin{tabular}{|c|c|c|c|c|c|}
\hline \multicolumn{2}{|c|}{ Animal } & \multicolumn{4}{|c|}{ Cooling time or Maneuver, (EMG* [times of test]) } \\
\hline No. & Side & $\begin{array}{l}5 \\
\text { seconds }\end{array}$ & $\begin{array}{l}3 \\
\text { seconds }\end{array}$ & $\begin{array}{l}\text { Immediately** } \\
\text { (with muscle } \\
\text { touch) }\end{array}$ & $\begin{array}{l}\text { Immediately** } \\
\text { (no muscle } \\
\text { touch) }\end{array}$ \\
\hline \multicolumn{6}{|c|}{ FMwand } \\
\hline \multirow[t]{2}{*}{7} & $\mathrm{~L}$ & Stable [3] & Stable [3] & - & Stable [3] \\
\hline & $\mathrm{R}$ & Stable [3] & Stable [3] & Stable [3] & - \\
\hline \multirow[t]{2}{*}{8} & $\mathrm{~L}$ & Stable [3] & Stable [3] & Stable [3] & - \\
\hline & $\mathrm{R}$ & Stable [3] & Stable [3] & - & Stable [3] \\
\hline \multirow[t]{2}{*}{9} & $\mathrm{~L}$ & Stable [3] & Stable [3] & - & Stable [3] \\
\hline & $\mathrm{R}$ & Stable [3] & Stable [3] & Stable [3] & - \\
\hline \multicolumn{6}{|c|}{ FMsealer } \\
\hline \multirow[t]{2}{*}{10} & L & Stable [3] & Stable [3] & - & $58.3 \%$ loss [1] \\
\hline & $\mathrm{R}$ & Stable [3] & Stable [3] & Stable [3] & - \\
\hline \multirow[t]{2}{*}{11} & $\mathrm{~L}$ & Stable [3] & Stable [3] & Stable [3] & - \\
\hline & $\mathrm{R}$ & Stable [3] & Stable [3] & - & LOS $^{+}[1]$ \\
\hline \multirow[t]{2}{*}{12} & $\mathrm{~L}$ & Stable [3] & Stable [3] & - & LOS' [1] \\
\hline & $\mathrm{R}$ & Stable [3] & Stable [3] & Stable [3] & - \\
\hline \multicolumn{6}{|c|}{$\begin{array}{l}\text { * EMG, electromyography, data of unstable column represents the final } \\
\text { amplitude after at least } 20 \text { minutes of continuously real-time EMG } \\
\text { recording. }\end{array}$} \\
\hline \multicolumn{6}{|c|}{$\begin{array}{l}{ }^{* *} \text { Estimate there is a 1-second time interval for shifting the device from } \\
\text { muscle to the RLN }\end{array}$} \\
\hline \multicolumn{6}{|c|}{$\begin{array}{l}\text { † LOS, loss of signal, defined as the evoked EMG amplitude less than } \\
100 \mu \mathrm{V}\end{array}$} \\
\hline
\end{tabular}

